\title{
THE FREDHOLM RADIUS OF A BUNDLE OF CLOSED LINEAR OPERATORS
}

\author{
E.-O. LIEBETRAU
}

\begin{abstract}
Given a bundle of linear operators $T-\lambda S$, where $T$ is closed and $S$ is bounded, a sequence $\left\{\delta_{m}(T: S)\right\}$ of extended real numbers is defined. If $T$ is a Fredholm operator, the $\operatorname{limit} \lim \delta_{m}(T: S)^{1 / m}$ exists and is equal to the supremum of all $r>0$ such that $T-\lambda S$ is a Fredholm operator for $|\lambda|<r$.
\end{abstract}

Throughout this paper $X$ and $Y$ are complex Banach spaces, $T$ is a closed linear operator with domain $D(T)$ in $X$ and range $R(T)$ in $Y$, and $S$ is a bounded linear operator from $X$ into $Y . K(X)$ is the space of compact linear operators on $X$, and $\Phi(T: S)$ is the set of those complex numbers $\lambda$ for which $T-\lambda S$ is a Fredholm operator.

Given $m \geqslant 1$, the element $\left(x_{1}, \ldots, x_{m}\right)$ of $D(T)^{m}$ is called a chain for $T$ and $S$ if $T x_{i}=S x_{i-1}$ for $i=2, \ldots, m$. Put

$$
\delta_{m}=\delta_{m}(T: S)=\sup _{C \in K(X)} \inf _{\left(x_{1}, \ldots, x_{m}\right)} \frac{\left\|T x_{1}\right\|}{\left\|(I-C) x_{m}\right\|},
$$

where the infimum is taken over all chains $\left(x_{1}, \ldots, x_{m}\right)$ for $T$ and $S$. Here $I$ denotes the identity mapping in $X$.

When $X=Y$ and $S=I$, the chains for $T$ and $S$ are of the form $\left(T^{m-1} x, \ldots, T x, x\right)$ with $x \in D\left(T^{m}\right)$, and

$$
\delta_{m}(T: I)=\delta_{1}\left(T^{m}: I\right)=\sup _{C \in K(X)} \inf _{x \in D(T)} \frac{\left\|T^{m} x\right\|}{\|(I-C) x\|} .
$$

Roughly speaking $\delta_{1}(T: I)$ is the reduced minimum modulus of $T$ corresponding to the $m$-seminorm introduced by A. Lebow and M. Schechter in [4]. $\delta_{1}(T: I)$ was studied in [5] and there it was shown that for a Fredholm operator $T, \lim \delta_{1}\left(T^{m}: I\right)^{1 / m}$ exists and is equal to the distance $d(0, \mathrm{C} \backslash$ $\Phi(T: I))$ of 0 to the complement of the Fredholm set of $T$.

THEOREM. Let $T$ be a Fredholm operator. Then

$$
\lim _{m \rightarrow \infty} \delta_{m}(T: S)^{1 / m}
$$

exists and is equal to $d(0, \mathrm{C} \backslash \Phi(T: S))$, the Fredholm radius of $T$ and $S$.

This result is closely related to the stability radius of a bundle of operators

Received by the editors June 29, 1977.

AMS (MOS) subject classifications (1970). Primary 47B30, 47A55; Secondary 47A10.

Key words and phrases. Fredholm operators, perturbation theory.

(1) American Mathematical Society 1978 
studied by H. Bart and D. C. Lay [1] in general and earlier by K.-H. Förster and M. A. Kaashoek [2] in the case $X=Y$ and $S=I$. In addition, the proof of the theorem requires a modification of the decomposition theorem of $T$. Kato [3, Theorem 4]. Both stability radius and decomposition use the following notation (see [3], [1]).

Define subspaces $N_{m}=N_{m}(T: S)$ and $R_{m}=R_{m}(T: S)$ of $X$ by

$$
\begin{array}{ll}
N_{0}=(0), & N_{m}=T^{-1} S N_{m-1}, \\
R_{0}=X, & R_{m}=S^{-1} T R_{m-1}, \quad m=1,2, \ldots
\end{array}
$$

The smallest number $m$ for which the null space $N(T)=N_{1}$ of $T$ is not contained in $R_{m}$ will be denoted by $\nu(T: S)$. Further let $\Delta(T: S)$ denote the open set of all $\lambda$ in $\Phi(T: S)$ such that $\nu(T-\lambda S: S)=\infty$.

1.1. Stability radius [1]. For $m \geqslant 1$ let $\gamma_{m}=\gamma_{m}(T: S)$ denote the supremum of all $c \geqslant 0$ with the property that

$$
\left\|T x_{1}\right\| \geqslant c \cdot d\left(x_{m}, N_{m}\right)
$$

for every chain $\left(x_{1}, \ldots, x_{m}\right)$. If $T$ is a Fredholm operator, the limit $\lim \gamma_{m}(T: S)^{1 / m}$ exists and is equal to $d(0, \mathbf{C} \backslash[\Delta(T: S) \cup\{0\}])$, i.e. the supremum of all $r>0$ such that $n(T-\lambda S)=\operatorname{dim} N(T-\lambda S)$ and $d(T-$ $\lambda S)=\operatorname{codim} R(T-\lambda S)$ are constant on $0<|\lambda|<r$.

1.2. Suppose $n(T)$ is finite. Then for $m \geqslant 1$ there is a compact projection $P_{m}$ of $X$ onto $N_{m}$ such that $\left\|P_{m}\right\| \leqslant m \cdot n(T)$. Then

$$
\left\|\left(I-P_{m}\right) x_{m}\right\| \leqslant\left\|I-P_{m}\right\| d\left(x_{m}, N_{m}\right) \leqslant\left\|I-P_{m}\right\| \gamma_{m}(T: S)^{-1}\left\|T x_{1}\right\|
$$

for every chain $\left(x_{1}, \ldots, x_{m}\right)$ where, as usual, $0^{-1}=\infty$. Therefore, $\gamma_{m}$ and $\delta_{m}$ are related by $\gamma_{m} \leqslant(1+m n(T)) \delta_{m}$.

2.1. Decomposition [3, Theorem 4]. Let $T$ be a Fredholm operator such that $\nu(T: S)$ is finite. There exist topological decompositions $X=X_{0} \oplus X_{1}$ and $Y=Y_{1} \oplus Y_{1}$ which completely reduce $T$ and $S$. For $i=0,1$, let $T_{i}$ and $S_{i}$ denote the restrictions of $T$ and $S$ to $X_{i}$ viewed as operators into $Y_{i}$. Then $\nu\left(T_{0}: S_{0}\right)=\infty, S_{1}$ is bijective, $S_{1}^{-1} T_{1}$ is nilpotent, and $\operatorname{dim} X_{1}=\operatorname{dim} Y_{1}<$ $\infty$. As a consequence, we have $\Delta\left(T_{0}: S_{0}\right)=\Delta(T: S) \cup\{0\}$.

2.2. Now take $0<\rho<d(0, \mathbf{C} \backslash(T: S))$ and let $\Delta_{\rho}$ denote the set of all complex numbers $\lambda$ such that $|\lambda| \leqslant \rho$ and $\nu(T-\lambda S: S)<\infty$. By induction we remove the finite set $\Delta_{\rho}$ from $\Delta(T: S)$ and obtain decompositions $X=X_{\rho}$ $\bigoplus X_{1}$ and $Y=Y_{\rho} \oplus Y_{1}$ such that $\Delta\left(T_{\rho}: S_{\rho}\right)=\Delta(T: S) \cup \Delta_{\rho}$, where $T_{\rho}$ and $S_{\rho}$ are restrictions of $T$ and $S$ to $X_{\rho}$ as in 2.1.

2.1 and 2.2 remain true if Fredholm operators are replaced by semi-Fredholm operators. However, this is not possible in the case 1.1 , see [1, 4.1].

Now we are able to prove the theorem. It will be shown

(a) $d(0, \mathbf{C} \backslash \Phi(T: S)) \leqslant \lim \inf \delta_{m}(T: S)^{1 / m}$ and

(b) $\lim \sup \delta_{m}(T: S)^{1 / m} \leqslant d(0, \mathrm{C} \backslash \Phi(T: S))$.

Both parts together establish the theorem.

(a) Since $T$ is Fredholm, $d(0, \mathrm{C} \backslash \Phi(T: S))$ is positive. Take $0<\rho<$ $d(0, \mathrm{C} \backslash \Phi(T: S)) .1 .1$ and 2.2 imply that $\rho \leqslant \lim \gamma_{m}\left(T_{\rho}: S_{\rho}\right)^{1 / m}$. For $m=$ 
$1,2, \ldots$ let $P_{m}$ be a projection of $X$ onto $N_{m}\left(T_{\rho}: S_{\rho}\right)$ with $\left\|P_{m}\right\|<m n\left(T_{\rho}\right)$ and let $\left(x_{1}, \ldots, x_{m}\right)$ be a chain for $T$ and $S$. Furthermore let $P$ and $Q$ be the bounded projections of $X$ onto $X_{1}$ along $X_{\rho}$ and of $Y$ onto $Y_{1}$ along $Y_{\rho}$, respectively. Then $P_{m} P=0, P_{m}+P \in K(X)$, and it is easy to verify that $\left((I-P) x_{1}, \ldots,(I-P) x_{m}\right)$ is a chain for $T_{\rho}$ and $S_{\rho}$. But then

$$
\begin{aligned}
\left\|\left[I-\left(P_{m}+P\right)\right] x_{m}\right\| & =\left\|\left(I-P_{m}\right)(I-P) x_{m}\right\| \\
& \leqslant\left\|I-P_{m}\right\| \gamma_{m}\left(T_{\rho}: S_{\rho}\right)^{-1}\left\|T_{\rho}(I-P) x_{1}\right\| \\
& \leqslant\left\|I-P_{m}\right\| \gamma_{m}\left(T_{\rho}: S_{\rho}\right)^{-1}\|I-Q\|\left\|T x_{1}\right\|,
\end{aligned}
$$

as in 1.2. Hence

$$
\left[\left(1+m n\left(T_{\rho}\right)\right)\|I-Q\|\right]^{-1} \gamma_{m}\left(T_{\rho}: S_{\rho}\right) \leqslant \delta_{m}(T: S),
$$

and consequently

$$
\rho \leqslant \lim \gamma_{m}\left(T_{\rho}: S_{\rho}\right)^{1 / m} \leqslant \lim \inf \delta_{m}(T: S)^{1 / m},
$$

which proves (a).

(b) Take $0<|\lambda|<\alpha<\lim \sup \delta_{m}(T: S)^{1 / m}$. First, suppose $\nu(T: S)=$ $\infty$. This restriction will be removed later with the aid of 2.1. It will be shown that $T-\lambda S$ is a Fredholm operator. There exists some $m$ and a compact operator $C=C_{\alpha, m}$ on $X$ such that

$$
\left\|(I-C) x_{m}\right\| \leqslant \alpha^{-m}\left\|T x_{1}\right\|
$$

for every chain $\left(x_{1}, \ldots, x_{m}\right)$ for $T$ and $S$. Since $T$ is Fredholm and $\nu(T: S)$ $=\infty$, that is, $N(T) \subseteq R_{m-1}$, there exists a relative inverse $L_{m}$ of $T$ such that $L_{m} T R_{n} \subseteq R_{n}$ for $n=0,1, \ldots, m-1$. Since $T R_{m-1}$ has finite deficiency in $Y$, there is a projection $Q$ of $Y$ onto $T R_{m-1}$ such that $\|Q\| \leqslant 1+m d(T)$. Take $y \in Y$ and put

$$
x_{i}=\left(L_{m} S\right)^{i-1} L_{m} Q y, \quad i=1, \ldots, m .
$$

$\left(x_{1}, \ldots, x_{m}\right)$ turns out to be a chain with $T x_{1}=Q y$. Consequently

$$
\left\|(I-C)\left(L_{m} S\right)^{m-1} L_{m} Q\right\| \leqslant \alpha^{-m}\|Q\|,
$$

and taking $Q=I-P, P \in K(Y)$, we have

$$
\left\|(I-C)\left(L_{m} S\right)^{m-1} L_{m}(I-P) S\right\|=\left\|\left(L_{m} S\right)^{m}-K_{m}\right\| \leqslant \alpha^{-m}\|Q\|\|S\|
$$

with some $K_{m} \in K(X)$.

Now let $\pi$ denote the canonical mapping from $B(X)$ onto $B(X) / K(X)$. Here $B(X)$ is the space of all bounded linear operators on $X$. Take any relative inverse $L$ of $T$. Then $L_{m}-L$ is degenerate, hence $\pi\left(L_{m} S\right)=\pi(L S)$, and the last inequality reads

$$
\left\|\pi(L S)^{m}\right\| \leqslant \alpha^{-m}(1+m d(T))\|S\| .
$$

This implies $r_{\sigma} \leqslant \alpha^{-1}<|\lambda|^{-1}$.

Here $r_{\sigma}$ is the spectral radius of $\pi(L S)$. But then $\lambda^{-1} \pi(I)-\pi(L S)$ is invertible in $B(X) / K(X)$, hence $I-\lambda L S$ is Fredholm and so is $T-\lambda T L S$. 
Since $L$ is a relative inverse of $T, T L=I-R$, where $R$ is a compact projection. So $T-\lambda S$ is a Fredholm operator if $\nu(T: S)=\infty$. Now suppose $\nu(T: S)$ is finite. Then $\nu\left(T_{0}: S_{0}\right)=\infty$ by 2.1. Let $P_{0}$ be the bounded projection of $X$ onto $X_{0}$ along $X_{1}$. Starting with a chain for $T_{0}$ and $S_{0}$ we obtain $\delta_{m}(T: S) \leqslant\left\|P_{0}\right\| \delta_{m}\left(T_{0}: S_{0}\right)$. Therefore

$$
0<|\lambda|<\lim \sup \delta_{m}(T: S)^{1 / m} \leqslant \lim \sup \delta_{m}\left(T_{0}: S_{0}\right)^{1 / m} \text {, }
$$

and by the preceding argument $T_{0}-\lambda S_{0}$ is Fredholm. Since $S_{1}^{-1} T_{1}$ is nilpotent and $\lambda \neq 0, T_{1}-\lambda S_{1}$ is bijective, thus $T-\lambda S$ is Fredholm. This proves (b).

COROLlary 1. Let $T$ be a Fredholm operator. Then $\Phi(T: S)=\mathrm{C}$ if and only if $\lim \delta_{m}^{1 / m}=\infty$, i.e. if and only if for each $\varepsilon>0$ and sufficiently large $m$ there are compact operators $C_{\varepsilon, m}$ on $X$, such that for every chain $\left(x_{1}, \ldots, x_{m}\right)$

$$
\left\|x_{m}\right\| \leqslant \varepsilon^{m}\left\|T x_{1}\right\|+\left\|C_{\varepsilon, m} x_{m}\right\| \text {. }
$$

Let $\hat{X}$ be $D(T)$ endowed with the graph norm $\|x\|_{T}=\|x\|+\|T x\|$, let $\hat{T}$ and $\hat{S}$ be the operators $T$ and $S$ considered as maps from $\hat{X}$ into $Y$, and let $i_{T}$ be the inclusion map of $\hat{X}$ into $X$. Then $\hat{X}$ is a Banach space, $\hat{T}, \hat{S}, i_{T}$ are bounded, and $\hat{T}=T i_{T}, \hat{S}=S i_{T}$. Put

$$
\hat{\delta}_{m}=\hat{\delta}_{m}(T: S)=\sup _{K \in K(\hat{X}, X)} \inf _{\left(x_{1}, \ldots, x_{m}\right)} \frac{\left\|\hat{T} x_{1}\right\|}{\left\|\left(i_{T}-K\right) x_{m}\right\|},
$$

where $\left(x_{1}, \ldots, x_{m}\right)$ is a chain for $\hat{T}$ and $\hat{S}$. Since $\hat{T} x_{1}=T x_{1}$ and $C \in K(X)$ implies $C i_{T} \in K(\hat{X}, X)$, we have $\delta_{m}(T: S) \leqslant \hat{\delta}_{m}(T: S)$.

Corollary 2. Let $T$ be a Fredholm operator. Then $\lim \hat{\delta}_{m}(T: S)^{1 / m}=$ $d(0, \mathrm{C} \backslash \Phi(T: S))$ and, as a consequence $\Phi(T: S)=\mathrm{C}$, if $i_{T}$ is compact.

Proof. By the preceding remark we have $d(0, \mathrm{C} \backslash \Phi(T: S)) \leqslant$ $\lim \inf \hat{\delta}_{m}^{1 / m}$. Replacing $T$ by $\hat{T}, S$ by $\hat{S}$, and $B(X) / K(X)$ by $B(\hat{X}, X) / K(\hat{X}, X)$ in part (b) of the theorem, we obtain lim sup $\hat{\delta}_{m}^{1 / m} \leqslant$ $d(0, \mathrm{C} \backslash \Phi(T: S))$. If $i_{T}$ is compact, then $\hat{\delta}_{m}=\infty$, hence the corollary.

Remark [5]. Let $X=Y$ be a complex Hilbert space, suppose $S=I$, and let $T$ be a densely defined normal Fredholm operator. Then $d(0, \mathbf{C} \backslash \Phi(T: I))=$ $\delta_{1}=\hat{\delta}_{1}$. If moreover $d(0, \mathbf{C} \backslash \Phi(T: I))<\infty$, i.e. the Fredholm set of $T$ is not the whole plane, then there exists a compact operator $K$ on $X$ such that $\delta_{1}(T: I)=\gamma(T-K)$, where $\gamma(T-K)$ denotes the reduced minimum modulus of $T-K$. These facts use the resolution of the identity corresponding to $T$.

\section{REFERENCES}

1. H. Bart and D. C. Lay, The stability radius of a bundle of closed linear operators, Univ. of Maryland, TR-76-11 (1976), 1-23.

2. K.-H. Förster and M. A. Kaashoek, The asymptotic behaviour of the reduced minimum modulus of a Fredholm operator, Proc. Amer. Math. Soc. 49 (1975), 123-131. 
3. T. Kato, Perturbation theory for nullity, deficiency and other related quantities of linear operators, J. Analyse Math. 6 (1958), 261-322.

4. A. Lebow and M. Schechter, Semigroups of operators and measures of noncompactness, $J$. Functional Analysis 7 (1971), 1-26.

5. E.-O. Liebetrau, Uber die Fredholmmenge linearer Operatoren, Dissertation, Dortmund, 1972.

Fachibbrich IV, Universitït Oldbnburg, D-2900 Oldenburo, Fedbral Rapubuc of GerMANY 